\title{
LA FRONTERA OCCIDENTAL DEL REINO DE MURCIA EN EL CONTEXTO DE LA INTERVENCIÓN ARAGONESA: DEFENSA Y REPOBLACIÓN (1270-1340)
}

\author{
Juan Francisco JIMÉNEZ ALCÁZAR \\ Universidad de Murcia
}

En el plazo aproximado de cinco décadas, desde la mitad del siglo XIII a los comienzos del XIV, el antiguo reino de Murcia, e incluso la tierra cercana a él, contempló su fragmentación de forma abrupta. Por el Poniente, la permanencia del poder nasrí y las circunstancias propias del proceso de conquista por el infante Alfonso derivaron en el trazo de una demarcación forzada en el centro de una gran comarca homogénea. Por el Levante, fueron las complejas relaciones políticas entre Aragón y Castilla, iniciadas un siglo atrás, las que decidieron su conformación definitiva!.

El establecimiento de Castilla en el Reino murciano se vio alterado bruscamente por la agresiva política de «castellanización" de Alfonso $X$. Tras tras la revuelta mudéjar de 1264-66, de forma inmediata se abrió el proceso repoblador en la mayor parte de las ciudades y villas de la demarcación y, paralelamente, el desarrollo institucional que organizaba comunidades y aparato militar. Así, la frontera con los nazaríes granadinos quedaba configurada en torno a unas tierras que dibujaban una línea perpendicular a la costa, partiendo desde las proximidades del puerto de Águilas hasta las defensas castellanas en Huéscar, Cúllar, Galera y Orce. Un punto centralizaba la red defensiva: la villa de Lorca, que además sostenía el paso a las tierras del reino de Murcia, constituido ya como un Adelantamiento, a través del valle del Guadalentín.

1 A pesar del tiempo transcurrido desde su publicación, continúa siendo de obligada referencia el estudio de TORRES FONTES, J., "La delimitación del Sureste peninsular (tratados de partición de la Reconquista)», Anales de la Universidad de Murcia, Curso 1950, pp. 5-32. 
Pero la planificación repobladora chocó con la ofensiva meriní y el inicio de lo que se viene denominando la «Batalla del Estrecho». Como bien es sabido, el proceso repoblador en las zonas más occidentales del reino se había caracterizado por la mayoría castellana, a diferencia de las ciudades orientales que lo fueron por pobladores procedentes de la Corona aragonesa. Prestaremos atención a este hecho cuando perfilemos la peculiaridad de la conquista de Jaime II. Por el momento, aludiremos a esta caracterización de mayoría castellana en la zona occidental por la presencia y configuración de la frontera con Granada. Después de la rebelión mudéjar y el desalojo de la plaza de posibles quintacolumnistas, la demarcación necesitaba, de forma inmediata, de un monto de población que asegurase la posición y la frontera, y no podía ser otro que el formado por contingentes dependientes directamente del soberano de Castilla. Además de este hecho, también hay que tener en cuenta otro tipo de factores interesantes, como la tradicional — que no desacertada - idea de que Jaime I no llegó hasta los límites con Granada y permaneció en las tierras orientales y centrales del reino, así como que Lorca era la plaza situada al Sur de las encomiendas santiaguistas, por lo que recibiría pobladores que continuaban el "circuito fronterizo" en busca de exenciones o sencillamente tierras.

De esta forma, una vez abierto el proceso repoblador en Lorca y con la subsiguiente articulación institucional castellana de la villa, se perfilaba una inflexión en el desarrollo histórico del territorioz ${ }^{2}$. El hecho en su conjunto no deja de tener una importancia capital en el sector, ya que Lorca se configuró como la plaza más decisiva para el mantenimiento fronterizo castellano. Lo había demostrado con la resistencia de Diego Sánchez de Bustamante en la fortaleza durante la rebelión mudéjar, pero con la ofensiva meriní a partir de la década de 1270 va a ser, si cabe, más definitiva. Es obvio que la firme presencia de los norteafricanos y el poder más asentado de los nazaríes colaboró en el afianzamiento de la línea fronteriza con Castilla. Por el momento nos interesa destacar que el mantenimiento granadino condicionó notablemente el desarrollo del reino murciano bajomedieval, ya que se esbozó como la cuña territorial de Castilla entre Aragón y Granada con ribera mediterránea. Este hecho repercutirá de forma intensa en las relaciones entre los tres reinos, debido a que Murcia se encontraba como testigo territorial de la mayor parte de sus contactos directos.

El reino de Murcia era una de las demarcaciones fronterizas de Castilla por antonomasia, marcado por la tan mencionada existencia de una triple frontera: con Granada, con Aragón y con la costa mediterránea. Pero desde el inicio de la repoblación, el sector occidental "sufrió» con mayor rigor la presencia de una frontera militar. La Corona castellana extendía directamente su poder en el sector más meridional de la línea, ya que había delegado la defensa de la zona Norte a las Órdenes del Temple y Santiago. Ésta última había estado avanzando por la Sierra de Segura hasta alcanzar Huéscar en 1241, y recibir un año después Orce y Galera, a la par que, años atrás, también había conseguido el arzobispo de Toledo, D. Rodrigo Jiménez de Rada, conquistar Quesada y buena parte de su entorno, definiendo una línea fronteriza a las puertas de Baza ${ }^{3}$. El poder

2 TORRES FONTES, J., Repartimiento de Lorca, Murcia, edic. 1994.

3 CARRIAZO ARROQUIA, J.M., Colección diplomática de Quesada, Jaén, 1975, pp. L-LIII. 
musulmán tenía en las plazas de Vera, Purchena y la ciudad bastetana los puntales defensivos más importantes, con pequeños puntos avanzados que, con el paso del tiempo, se fueron configurando como la vanguardia defensiva nazarí; es el caso de Huércal y fundamentalmente los Vélez.

En plena etapa de caos político castellano, zenetes veratenses lograron asestar un durísimo golpe a los planes repobladores cristianos; en 1283, una cabalgada en el campo lorquino logró dar muerte a doscientas personas y hacer cautivas a otras tantas. Ya había comenzado el repliegue poblacional del entorno rural al más seguro de las defensas urbanas. Se potenciaba ahora el fenómeno de las despoblaciones, iniciado décadas atrás con el desalojo de los mudéjares de los sectores fronterizos. La cabalgada de 1283 no hizo sino potenciar este proceso. Por aquellos años se asistía a la virulencia de una vida fronteriza muy activa, con golpes de mano, algaras y celadas continuas, y que ya definía el campo como un lugar potencialmente peligroso; por ejemplo, una travesía entre villas podía convertirse en toda una desventura, como en efecto lo fue para Ramón de Mula, cautivado en 1285 cuando se desplazaba desde Lorca hasta Mula ${ }^{4}$.

La única respuesta de los repobladores, articulados ya en concejos y con usos fronterizos defensivos - caso de la obligación de los caballeros villanos de mantener caballo y armas ${ }^{5}$, fue el abandono de la zona o el repliegue a lugares seguros. El ejemplo más conocido es el del obispo de Cartagena que se estableció oficialmente en 1291 en la capital del reino. En los albores de la intervención aragonesa podemos decir que la mayor parte de las zonas cultivadas del sector fronterizo con Granada se habían abandonado, quedando exclusivamente las estrechas franjas de vega en villas como Mula y Lorca como espacios productivos. Incluso se llegaron a desalojar núcleos más pequeños, como Puentes, el enclave más avanzado castellano hacia las tierras orientales nazaries. En esos años, el rey granadino había conseguido recuperar Quesada - 12956 - hasta entonces en poder del arzobispado toledano, y que abría una etapa agresiva de Granada contra Castilla, por la firma de paces entre Jaime II y Muhammad II. Este es el contexto fronterizo en donde hemos de situar el privilegio de 1295, ya con Fernando IV, para que con el sietmo de las cabalgadas se reforzasen las defensas de la villa de Lorca ${ }^{7}$.

La minoría de Fernando IV abrió una época de indolencia monárquíca en Castilla, donde las hermandades hallaron de nuevo un período floreciente. En el reino de Murcia se firmó una hermandad general en octubre de 1295 entre las principales ciudades y villas ${ }^{8}$, y en ella, como no podía ser de otra manera, se garantizaba el señorío de

4 TORRES FONTES, J., Repartimiento de Lorca, pp. 53-54.

5 GONZÁLEZ JIMÉNEZ, M., "Alfonso X y las oligarquías urbanas de caballeros", Glossae, 5-6 (1993-94), p. 200.

6 CARRIAZO ARROQUIA, J.M., ob. cit., pp. LXVIII y ss.

7 TORRES FONTES, J., Documentos de Fernando IV, CODOM V, Murcia, 1980, p. 19.

8 BENAVIDES, A., Memorias de D. Fernando IV de Castilla. Tomo II, RAH, Madrid, 1860, pp. 4650. Al final, hay una nota de Diego Clemencín justificando el espacio en blanco que aparece en el documento, destinado para Orihuela. También se incorporó Cartagena después de formalizada la hermandad: p. 52. 
Fernando IV en tierras murcianas, capítulos equiparables a las firmadas de forma paralela en León y Galicia. De parte lorquina, firmaron Diego Álvarez de Espejo, el adalid Pedro Juan de las Cuevas y Alonso Fernández de la Torre ${ }^{9}$. Poco sospechaban que la amenaza procedería de Aragón tan sólo meses más tarde, y no de Granada ni del interior.

En efecto, los últimos años del siglo XIII vieron que el interés mediterráneo de Jaime II se prolongaba por las tierras murcianas. Para un enfrentamiento abierto con los castellanos era preciso establecer buenas relaciones con los granadinos, acuerdo que debía incluir que los musulmanes actuasen militarmente en la frontera andaluza. Los beneficios para los aragoneses estaban claros, pues se realizaba una maniobra de distracción de contingentes castellanos que pudieran participar en el escenario murciano o en la frontera aragonesa; la ventaja para los nazaríes era que se aseguraba nuevamente la supervivencia del reino granadino. La firma del acuerdo entre Jaime II y Muhammad II en mayo de $1296^{10}$, halla en este contexto su explicación más certera.

Con los aragoneses ya en Murcia, los puntos más occidentales del reino veían que su labor defensiva tenía sentido no sólo contra los nazaríes, sino también contra los aragoneses. Desde la capital, tenemos noticias de que Jaime II envió a un franciscano al concejo de Lorca ${ }^{11}$, sospechamos que con la intención de preparar una capitulación de la villa. Mucho más directo fue el emplazamiento del rey aragonés a Juan Fernández, alcaide del castillo de Chuecos ${ }^{12}$, defensa enclavada entre las sierras de Almenara y Carrasquilla, y que, junto a Tébar, dominaba el único paso accesible desde el puerto costero de Águilas al valle del Guadalentín. En mayo de 1296, Mula había caído en manos aragonesas, aunque Alhama, al pie de Sierra Espuña, aún resistía. Por aquellos mismos días, Jaime II acogía bajo su protección las villas de Cehegín y Caravaca, pertenecientes al Temple ${ }^{13}$, por lo que entendemos que existió un acuerdo en la capitulación pacífica. A pesar de esta capitulación, no vamos a reincidir en la importancia que se le ha dado a la peculiaridad repobladora de esta zona, con la mayoría castellana ya aludida y que llega a mencionar la propia Crónica de Fernando $\mathrm{I}^{4}$.

9 Ibídem, p. 45.

10 Del ESTAL, J.M., El reino de Murcia bajo Aragón (1296-1305). Corpus documental I/1, Alicante, 1985, pp. 164-166.

11 Ibídem, p. 191.

12 Ibídem, pp. 192-93.

13 Del ESTAL, J.M., El reino de Murcia bajo Aragón (1296-1305). Corpus documental 1/2. Alicante, 1990, pp. 59-60. Sobre la presencia templaria en la bailía de Caravaca: PASCUAL MARTÍNEZ, L., «Los templarios en el Reino de Murcia», Homenaje al prof. Muñoz Cortés, t. II, Univ. Murcia, 1977, pp. 871-886.

14 Crónica de Fernando IV, edic. de BENAVIDES, A., en: Memorias de Fernando IV de Castilla, tomo I, Madrid, RAH, 1860, pp. 1-243. En la edición de la BAE (Crónicas de los reyes de Castilla, tomo I, Madrid, 1953) en pp. 91-170. 
«E por consejo de los de la tierra, que eran catalanes, dieronsele todas las villas e los castillos, salvo ende Lorca, en que moraban castellanos e otrosi Alcala [Puebla de Mula ${ }^{15}$ e Mula».

Su interés radica en la confirmación y aceptación de unos hechos por parte castellana que, por otro lado y para los fines propagandísticos de la crónica, venían a crear una causa razonable que eximiera a la Corona castellana del fracaso militar y repoblador en el Reino de Murcia, culpando a los propios habitantes de la demarcación.

A principios de junio, Jaime II se encontraba a las puertas de Lorca ${ }^{16}$. El sistema logístico escogido había sido el de apoyo y abastecimiento por mar, con el desembarco en el puerto de Águilas ${ }^{17}$. En estas maniobras observamos la verdadera dimensión de la tregua firmada dos semanas atrás entre Jaime II y Muhammad II, ya que la tregua con los granadinos aseguraba las operaciones marítimas catalanas, sobre todo en latitudes tan cercanas a las tierras nazaríes, y fundamentalmente garantizaba la quietud granadina durante los movimientos terrestres. El fracaso de lo que fue el primer cerco de la plaza lorquina se explica por tres motivos principalmente: por la inexpugnabilidad de las defensas, que procuraban un esfuerzo militar mucho mayor del que había movilizado Jaime II en esta ocasión; en segundo lugar, porque existían problemas logísticos muy complejos, ya que debía mantener lo conquistado a retaguardia; y tercero y consecuencia del anterior, porque estaba asegurando esas conquistas «despejando» los núcleos resistentes, con órdenes de confiscación y expulsión a los que habían huido o resistido ${ }^{18}$.

Las líneas maestras del juego diplomático entre Aragón y Granada condicionaron buena parte de los sucesos fronterizos realizados en este sector. La confirmación de la paz catalano-granadina en septiembre de $1296^{19}$ significaba un mantenimiento de la situación generada tras la invasión en Murcia, intenciones reafirmadas con los contactos de junio del año siguiente entre ambas Coronas, pretendiendo además Jaime II que el sultán nazarí reconociese a Alfonso de la Cerda como rey de Castilla ${ }^{20}$.

Las consecuencias de la intervención aragonesa en el reino murciano no se hicie-

15 Queda demostrada su localización por GONZÁLEZ CASTAÑO, J., Una villa del Reino de Murcia en la Edad Moderna (Mula, 1500-1648), Murcia, 1992.

16 El 4 de junio de 1296 expide una carta desde la huerta de Lorca. Del ESTAL, J.M., El reino de Murcia bajo Aragón (1296-1305). Corpus documental 1/2, p. 60.

17 El 24 de mayo, Jaime II había escrito a su consejero Bernardo de Sarriá indicándole su intención de marchar sobre Lorca dos días después, y con el encargo de que enviase vituallas a Águilas. Ibídem, pp. 56-57.

18 A partir de estas fechas se documentan en el A.C.A., muchas de ellas recogidas en los corpus documentales publicados por J.M. Del Estal, una cantidad notable de cartas de confiscación de bienes a resistentes y otorgamiento de bienes a leales o colaboracionistas.

19 Posiblemente se trate de los mismos capítulos del tratado de mayo. 1296-IX-8. Carta de Jaime II al rey de Granada, diciéndole que ha recibido el tratado de paz ajustado entre los dos, y aplazado para otra época la conclusión de los asuntos pendientes. BENAVIDES, A., Memorias de D. Fernando IV de Castilla, pp. 98-99.

20 Ibídem, p. 124. 
ron esperar, y la más importante, sin duda alguna, fue la anulación de la presencia castellana en el Mediterráneo. La repercusión de ęste hecho en la guerra abierta por el Estrecho era bastante notable para Castilla. Además, la presión iniciada años atrás por nazaríes y meriníes hizo que a la caída de Quesada se le unieran Alcaudete -1300-, Bedmar, Arenas y Locubín -130221 - En el sector murciano, el dominio castellano se seguía restringiendo a Lorca, que permanecía aislada, ya que entre Alcaraz y la villa del Guadalentín, las tropas aragonesas controlaban la bailía de Caravaca. Mula permaneció tan sólo dos años bajo poder aragonés, pues en 1298 la villa se levantó y volvió al señorío de Fernando IV ${ }^{22}$. La caída de Alhama ese mismo año complicó la situación militar del sector, lo que no desahogó la posición lorquina.

Durante cuatro años, la villa lorquina resistió diversos embates aragoneses, por lo que recibió de la Corona dos de los privilegios más conocidos en la Historia de la ciudad. El primero de ellos fue el de acuñación de moneda, concedido en Toro en $1297^{23}$. Las razones de esta concesión las especifica claramente el propio documento: a causa de la guerra que mantenía el rey con el infante $D$. Juan, aspirante al trono D. Alfonso de la Cerda, y de forma fundamental, porque la villa de Lorca esta mucho afincada de guerra de los christianos [aragoneses] et de los moros. No era la primera vez que ante la coyuntura bélica, la Corona delegase esta regalía. Desde el punto de vista estrictamente práctico, la concesión era lógica, ya que Lorca se encontraba aislada, pero también se escondían otras causas relacionadas con motivos económicos más profundos. La moneda que se había ordenado labrar era de «bajísima ley», de dos dineros el maravedí, y pretendía sustituir a la acuñada por Sancho IV (el privilegio menciona incluso la compra de esa moneda para la confección de la nueva), que era de mejor calidad, en un sencillo desarrollo de sustitución de la moneda buena por la mala ${ }^{24}$. Con esta operación se quería evitar la fuga de moneda, a la vez que una medida

21 LADERO QUESADA, M.A., Granada. Historia de un país islámico, Madrid, 1989, p. 144.

22 El 8 de mayo de 1298, Jaime II se dirigió a Jaime de Jérica, Procurador de Murcia, ordenándole recobrar Mula. BENAVIDES, A., Memorias de D. Fernando..., pp. 163-164. De parte castellana, a comienzos de julio, Fernando IV confirmó sus privilegios: TORRES FONTES, J., Documentos de Fernando IV, CODOM V, Murcia, 1980, p. 30. Es interesante observar el papel que en esta sublevación tuvo el comendador santiaguista de Ricote, Fernando Pérez, en respuesta a los términos recriminatorios contenidos en una carta de Jaime II a Juan Osórez como maestre de Santiago a finales de 1300, durante el cerco de Lorca ["que Ferran Perez comendador de la dita val acuylle e recibe nuestros enemigos en los castiellos de la orden e con complio esto fizo nos perder el castiello de Mula"]: Del ESTAL, J.M., El Reino de Murcia bajo Aragón, 1/2, p. 131.

23 Archivo Municipal de Lorca. Lo transcribió ESPÍN RAEL, J., en: Privilegio para labrar moneda en Lorca, dado en Toro, por el rey Fernando IV, en 24 de octubre de la Era de 1335, Lorca, 1936. A raíz de la transcripción del erudito local, con bastantes lagunas, realizó un interesante estudio numismático GIL FARRÉS, O., «En torno del privilegio de Lorca y distinción entre las monedas de vellón de Fernando III y de Fernando IV", Numario Hispánico, V (1956), pp. 263-279. No obstante, el privilegio fue publicado de forma íntegra por TORRES FONTES, J., en: Documentos de Fernando $\mathrm{N}$, pp. 26-30, transcripción que hemos utilizado. Analiza las monedas procedentes de esta acuñación FONTELA BALLESTA, S., Las acuñaciones medievales de Lorca, Lorca, 1995, pp. 61-63.

24 GIL FARRÉS, O., ob. cit., p. 266. 
devaluadora respondía a las necesidades perentorias de liquidez por parte de la Corona. El hecho ayudaba además a mantener en circulación moneda castellana, ya que meses atrás Jaime II había dado licencia para labrar moneda en Alicante ${ }^{25}$.

El segundo de estos privilegios, y mucho más conocido, fue el de 1299. En él, la Corona concedía a la villa diversos castillos del entorno territorial: Caristoy, Calenque, Ugéjar, Amín, Nogalte, Puentes, Celda y Coy ${ }^{26}$. Lo pretendido fue reforzar el sistema defensivo del sector con la centralización del poder militar; no obstante, la desorientación de la Corona era notable, ya que Puentes pertenecía a la jurisdicción de la villa desde la temprana concesión de $1257^{27}$, y Alhama había caído en manos aragonesas hacía un año. Diferente es el caso de $\operatorname{Coy}^{28}$, ya que tras la muerte de su señor, Nicolás Pérez, en 1296 defendiendo la fortaleza alicantina, el enclave creemos que revirtió al patrimonio real.

Cabe ahora preguntarse la efectividad de esta concesión, en primer lugar porque la fortaleza lorquina cayó en manos aragonesas al año siguiente, con lo que se alteraba la ordenación territorial; y segundo, por las consecuencias que se observan con posterioridad al regreso de Lorca a la Corona castellana. El dominio aragonés apenas tuvo consecuencias inmediatas, debido al relativamente corto espacio de tiempo que duró la ocupación - cuatro años-, más cuando hemos de tener en cuenta las condiciones de la capitulación pactada en aquella Navidad de 1300, donde Jaime II respetaba privilegios, fueros y libertades de la villa y concejo; la conclusión más obvia hubiera sido que este privilegio también se hallase entre esas condiciones. Pero en 1304, tras Torrellas, Lorca volvió a manos castellanas. Con el pendón castellano ondeando nuevamente en la fortaleza de la villa, la efectividad del privilegio es muy dudable. Según el perdón de Fernando IV a los vecinos de Lorca, los privilegios se confirmaron, por lo que deberíamos tener en cuenta otra nueva aceptación del privilegio por las autoridades castellanas. Pero sabemos de su inefectividad ya en las primeras décadas del XIV. El profesor Torres Fontes apoya la hipótesis de que fue la posición política de D. Juan Manuel en 1320-30 la que finalmente deshizo la posible realidad del privilegio ${ }^{29}$, opinión que compartimos.

La efectividad más importante del documento fue que en éste se halló la base legal y documental para expandir el alfoz, durante el proceso de ampliación del término jurisdiccional a lo largo de la Baja Edad Media. Por ello, no sorprende su mención y utilización en diversos pleitos muchos años después, ya en los años postreros de la Edad Media. Adquirió otra dimensión a partir del siglo XVII, donde el documento era

25 Del ESTAL, J.M., El Reino de Murcia bajo Aragón, 1/2, pp. 77-79.

26 A.M.L. Pergamino. Publicado por TORRES FONTES, J., en: Repartimiento de Lorca, Murcia, edición de 1977, doc. XXIV, pp. 94-95. Analicé el documento en el amplio contexto de la expansión del alfoz concejil lorquino en: Lorca: ciudad y término (ss. XIII-XVI), Murcia, 1994, pp. 120-121.

27 TORRES FONTES, J., Fueros y privilegios de Alfonso $X$ al Reino de Murcia, CODOM III, Murcia, 1973, pp. 41-43.

28 JIMÉNEZ ALCÁZAR, J.F., «Tierra, propiedad y paisaje agrario en la frontera de Granada: Coy (Lorca, Murcia)", Anales de la Universidad de Alicante, 10 (1994-1995), pp. 169-195.

29 TORRES FONTES, J., Repartimiento de Lorca, Murcia, 1994, p. LXXXIV. 
-como lo es hoy— la enseña y «caballo de batalla» para cronicones y eruditos locales, que cantaron - y cantan- glorias pasadas de la ciudad.

En estos años, la clave de la resistencia lorquina se centraba en la doble vertiente fronteriza que encarnaba la villa y territorio; por un lado la costa, convertida en el único resquicio por el que Castilla se asomaba al Mediterráneo, si bien es cierto que de forma testimonial; y por otro, la frontera terrestre con Granada. Este hecho no es menos importante, ya que era fundamental para los planes expansivos peninsulares de las Coronas aragonesa y castellana.

No vamos a entrar en los sucesos concretos de la capitulación lorquina ${ }^{30}$, sino más bien en el contexto en que se hizo. En primer lugar, hay que tener en cuenta la paz firmada entre Aragón y Granada en febrero de $1300^{31}$, viviendo aún Muhammad II. De ahí que entendamos perfectamente la quietud de las huestes musulmanas en las vísperas del cerco definitivo a Lorca, más cuando en el resto de la frontera castellanogranadina, los nazaríes estaban realizando una presión militar importante, sobre todo en la zona jiennense ${ }^{32}$, cuyo fruto más evidente fue la reconquista granadina de la plaza de Alcaudete.

Para entender perfectamente la caída de Lorca durante el cerco de la Navidad de 1300 , hemos de tener en cuenta dos motivos principales: el papel jugado por D. Juan Manuel, que a la sazón se encontraba refugiado entre los muros de la fortaleza, y, sobre todo, la decisión de los poderosos dominantes del concejo, que al fin y a la postre, aceptaron las condiciones de capitulación ofrecidas por Jaime II. Entre los firmantes podemos observar a individuos pertenecientes a linajes importantes de la villa, como Teruel, Pérez de Tudela, Navarro, Pérez de Meca o Morata ${ }^{33}$. En la confirmación de fueros y libertades dado por Jaime Il días después de la cesión de la villa, se contemplaba la atención de la Corona para con los de cavallo ${ }^{34}$, por lo que hemos de tener muy presente la actuación del grupo dominante, aún no definido con precisión, en la capitulación lorquina. La hipótesis parece confirmarse a tenor de las protestas que realizó la ciudad de Orihuela tres años después, por no gozar los caballeros oriolanos el mismo trato de favor que tenían los de Lorca y Murcia ${ }^{35}$. También hay que suponer otro hecho no contemplado en los documentos, y es la perspectiva ofrecida a los vecinos de una futura pertenencia a Aragón. Cierto es que en la villa se dejó sentir rápidamente la concordia granadino-aragonesa, ya que la firma de relaciones

30 Las noticias documentales precisas fueron publicadas por Del ESTAL, J.M., en: «Nuevos datos sobre la capitulación y conquista aragonesa de Lorca», Homenaje al prof. Torres Fontes, vol. I, Univ, Murcia, 1987, pp. 431-464.

31 BENAVIDES, A., Memorias de D. Fernando IV.., pp. 203-205.

32 GONZÁLEZ MÍNGUEZ, C., Fernando IV (1295-1312), Palencia, 1995, p. 65.

33 Del ESTAL, J.M., «Nuevos datos...", pp. 442-447. A Martín de Morata lo podemos ver incluso entre los caballeros alforrats: FERRER I MALLOL, M.T., Organització i defensa d'un territori fronterer. La governació d'Oriola en el segle XIV, Barcelona, CSIC, 1990, pp. 359-361.

34 1300-XII-29, Lorca. Confirmación por Jaime II de los fueros que disfrutaba la villa de Lorca hasta la fecha de su rendición: Del ESTAL, J.M., El Reino de Murcia bajo Aragón, 1/2, pp. 143-44.

35 FERRER I MALLOL, M.T., Organització i defensa..., pp. 352-353. 
comerciales entre ambas coronas en abril de $1301^{36}$, llevaba parejo el paso inevitable de toda afluencia mercantil por Lorca como paso natural.

Con referencia a D. Juan Manuel, es posible que su papel quedase mediatizado o al menos ensombrecido por el protagonismo de los vecinos. Será años más tarde, cuando el autor del Conde Lucanor se «enseñorée» de la villa.

Tras la capitulación de Lorca y la previa caída de Calasparra, Alcaraz se convirtió en la villa-base de frontera del sector Sureste de Castilla frente a Granada, con Mula como vanguardia castellana sobre el núcleo del reino, y Moratalla compartiendo frontera con Aragón -bailía de Caravaca-y Granada. La villa de Lorca, ya bajo dominio aragonés, continuaba manteniendo una doble frontera terrestre, ya que mantenía la granadina y se generaba la septentrional con la vanguardia castellana situada en Mula. Esta villa se convirtió en punto de hostigamiento constante tanto para las tierras bajo dominio aragonés como las de los nazaries; de ello es buena muestra la petición de Muhammad II a Jaime II de que castigase a Mula por las continuas correrías que realizaban sus vecinos en las zonas orientales granadinas ${ }^{37}$. Lo cierto es que el ambiente de guerra en el reino estaba generalizado, con celadas y algaras constantes por parte de unos y otros ${ }^{38}$.

La estructuración defensiva de la frontera aragonesa con Granada en la zona murciana repetía el sistema castellano, fundamentándose en dos puntos principales: el eje Cahegín-Caravaca al Norte, y Lorca dominando el gran valle de acceso al Levante. El mantenimiento de este esquema defensivo era inevitable y fue llevado a cabo de forma inmediata, habida cuenta que se prolongaba la guerra con Castilla y que se quiso invertir en infraestructuras defensivas y guarnición de Lorca, compuesta en 1303 por cien hombres ${ }^{39}$. En ese mismo año, y coincidiendo con la presencia constante en la villa de Pedro de Monteagudo, Procurador General del Reino, lo que demuestra la importancia estratégica de la plaza, se alude a la necesidad de realizar dos grandes obras en el recinto de la fortaleza, en concreto dos aljibes en las torres Alfonsina y Espolón ${ }^{40}$.

Pero el núcleo principal se asentaba sobre otros enclaves menores, que tejían la tupida red defensiva, como el de Aguaderas, situado también en el valle, y que ya había sido reforzado en tiempos del cerco a Lorca ${ }^{41}$; en este mismo plano se situaban los castillos de Chuecos y Tébar, que capitularon a los pocos días de haber caído la villa lorquina, y que seguirán activos, con una guarnición de 12 hombres.

La utilidad del sistema defensivo se evidenció cuando el sector retomó su

36 MASIÁ, A., Jaume Il: Aragó, Granada i Marroc, Barcelona, CSIC, 1989, pp. 118-121.

37 Ibídem, p. 198.

38 Se documentan incluso contingentes musulmanes al servicio de Jaime II que hostigaban toda la tierra circundante que no estaba bajo dominio aragonés, es decir, tierra castellana y también granadina: MASIÁ, M.A., Relación castellano-aragonesa desde Jaime I/ a Pedro el Ceremonioso, vol. I, Barcelona, 1994, p. 144.

39 FERRER I MALLOL, M.T., Organització i defensa..., p. 192.

40 MASIÁ, M.A., Jaume II: Aragó, Granada i Marroc, p. 79.

41 FERRER I MALLOL, M.T., Organització i defensa..., pp. 28-31. 
protagonismo frente a la frontera occidental. La firma de la tregua entre Castilla y Granada en septiembre de 1303 infundió preocupación en las demarcaciones más próximas a la línea ${ }^{42}$, a pesar de las primeras intenciones nazaríes de mantener la tregua con los aragoneses ${ }^{43}$.

Sin embargo, las negociaciones castellano-aragonesas dieron resultado y el acuerdo de Torrellas (8-VIII-1304) precipitó que tan sólo dos días después, la Cancillería de Fernando IV expidiese perdones para Murcia, Lorca, Alhama y Molina ${ }^{44}$. En 1305, este perdón fue confirmado por el rey castellano. Este hecho respondía a la política que se pretendía realizar, de status quo por parte del nuevo adelantado del reino de Murcia, D. Juan Osórez. Lorca sería devuelta a Castilla el 21 de noviembre, la última de toda la serie de devoluciones; la razón no es otra sino que geográficamente era la más lejana de la ruta seguida por el maestre santiaguista y sus mandatarios castellanos.

La Corona castellana retomó el interés por mantener activas las defensas con Granada, aunque de diferente forma a la aragonesa, ya que si ésta había sido de inversión directa, Fernando IV otorgará un nuevo privilegio con el fin de que se autosufragasen las costosas obras ${ }^{45}$. La escasa utilidad de este privilegio va acompañada del fracaso repoblador, que se acentuó tras la ocupación aragonesa. Un buen indicio de este hecho lo ofrece otro privilegio del Emplazado; después de su perdón, dio licencia para reocupar las heredades de los ausentes ${ }^{46}$, lo que significa un proceso de abandono poblacional, desde aquellos que marcharon al llegar los aragoneses, hasta los que huyeron al recuperar la plaza la Corona castellana.

Las relaciones de Castilla con Granada pasarán en los años siguientes por la participación aragonesa, encauzada a través del tratado de Alcalá de Henares. La conquista de Lubrín por el obispo de Cartagena, o la fallida empresa almeriense de Jaime II, fueron acciones ofensivas que no apagaron la presión nazarí en los años siguientes a 1310. Fue precisamente este empuje fronterizo el que proporcionó una nueva configuración de la línea en las dos décadas siguientes, ya que la zona norte santiaguista será reconquistada por los granadinos.

Las treguas de 1310-12 dieron el primer aldabonazo para esa conformación definitiva aludida para este sector, ya que mientras la zona de Cazorla quedó para Castilla, todo el sector sur tornó a manos nazaríes. En esta zona, quedó establecido institucionalmente el arzobispado de Toledo a través del adelantamiento de Cazorla ${ }^{47}$, articulado en una demarcación fronteriza que se extenderá hasta los momentos de la conquista de Baza en 1489. La conquista de Cambil, Alhabar, Belmez -1316 a 1317-y de Tíscar en 1319

42 MASIÁ, M.A., Jaume II: Aragó, Granada i Marroc, pp. 79-81.

43 Ibídem, p. 78.

44 TORRES FONTES, J., Documentos de Fernando $N$, p. 33, quien recoge también la confirmación de la carta de perdón al concejo de Lorca: p. 45.

45 lbídem, pp. 46-47.

46 Ibídem, p. 48.

47 GARCÍA GUZMÁN, M.M., El Adelantamiento de Cazorla en la Baja Edad Media, Univ. Cádiz, 1985. 
no pudo paliar el desastroso balance en la zona jiennense tras la muerte de los infantes regentes $D$. Juan y $D$. Pedro durante una cabalgada a la Vega granadina.

La otra célula fronteriza, fundamental para la conexión del sector más oriental de la frontera con el núcleo de Castilla, es decir, la zona centralizada por Lorca, estaba controlada por Santiago. Desde que en 1241 Huéscar hubiese caído en manos santiaguistas y al año siguiente, otorgadas a la citada orden las villas de Galera y Orce, todo el sector sufrió una presión muy intensa, ya que abría las puertas de una zona económicamente muy rica en pastizales de invierno. La reacción nazarí tras el desastre de los infantes provocó que en 1324 ismail reconquistara la villa oscense ${ }^{48}$, con el empleo de un ingenio artillero.

La caída de tal enclave provocó una reestructuración de todo el sistema fronterizo, y el planteamiento de la realidad defensiva era procedente para mantener esa estructura. Esta es la explicación más evidente para explicar el nuevo proyecto de repoblación del campo lorquino en 1336, en vísperas del encuentro definitivo en el Salado.

El aspecto del campo lorquino en la primera mitad del XIV era bastante desolador, y si concebimos la frontera como una línea esencialmente humana, la mantenida por Castilla en el sector oriental era de un nivel poblacional muy débil. Por ello, se concibió un nuevo proyecto de repoblación en 1330, paralizado varios años por las maniobras políticas de D. Juan Manue ${ }^{49}$, y que pretendía dar un nuevo impulso a la instalación de contingentes en la villa. Tras el Salado y la victoria de Sancho Manuel en Velillas -1341-, la frontera se aquietó en las décadas siguientes, no alterada territorialmente hasta la campaña de 1406. Veremos que la actividad fronteriza se concentró en el Norte y Oeste de la línea fronteriza, lo que correspondía a la aludida Banda Morisca de los reinos andaluces. Es la época de la conquista de Alcalá de Benzaide —Alcalá la Real-, y la recuperación de Alcaudete, y de los sitios de las plazas del Estrecho.

Concluyamos diciendo que el sector fronterizo se forjó con las convulsiones interiores más que directas, ya que hemos podido comprobar que no hubo grandes operaciones fronterizas que repercutiesen en cambios sustanciales en la frontera, si exceptuamos la recuperación de Huéscar por Ismail. El proceso repoblador resultó un fracaso, por lo que no hemos de achacar únicamente al desarrollo pestilencial de 1348 como protagonista de la despoblación fronteriza. Es muy significativa la descripción que dio Cánovas Cobeño de la villa de Lorca para estos duros años de implantación castellana y asentamiento fronterizo con Granada; para él, Lorca quedó «reducida a la condición, no de una población regular, sino de una plaza o presidio militar fortificado", realidad que hemos de extender para el resto de núcleos que se mantuvieron habitados.

48 LADERO QUESADA, M.A., Granada..., p. 148.

49 TORRES FONTES, J., Repartimiento de Lorca, 1994, pp. LXXXVI-XC. 